\title{
Organocatalytic Vinylogous Michael Additions
}

Key words

vinylogous Michael addition

quinines

dienamine catalysis

primary amines

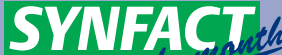

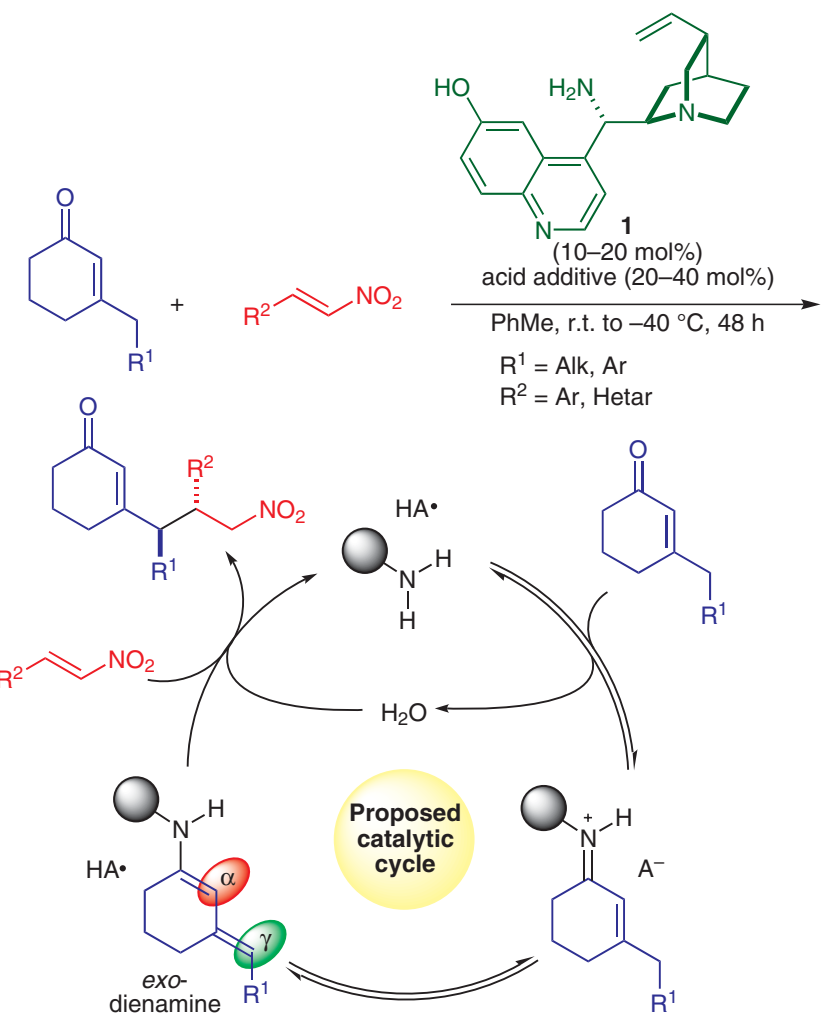
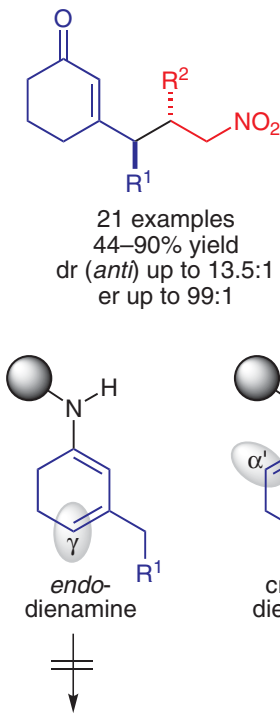

not observed

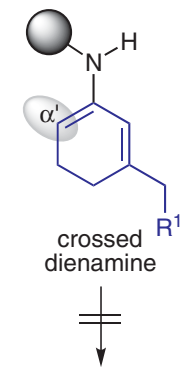

not observed
Significance: The catalytic enantio-, diastereoand $\gamma$-selective Michael addition of $\beta$-substituted cyclohexenones to nitroalkenes and nitrostyrenes is reported. The catalytic concept relies on the dienamine formation of amino salt $\mathbf{1}$ with the carbonyl compound, enabling nucleophilic attack on the nitroalkene from the exo- $\gamma$ position. Possible side products arising from an endo- $\gamma$ or crossed dienamine $\alpha$-attack to the electrophile are not observed. Furthermore, the reported method enabled the construction of quaternary stereocenters since $\beta, \beta$-disubstituted nitrostyrenes and $\alpha$-methyl cinnamates could be transformed into the corresponding Michael products equally well.
Comment: The appealing concept of vinylogy is rarely addressed in organocatalysis. Seminal reports dienamine catalysis by Jørgensen and coworkers (J. Am. Chem. Soc. 2006, 128, 12973) describe the $\gamma$-amination of enals. In the present work Melchiorre and co-workers significantly enlarge the scope of vinylogous organocatalysis by using an interesting though somewhat specialized substrate class as nucleophile precursor for which notably other envisionable dienamine scenarios seem to be negligible. However, further research to extend the scope and applicability of the method and to elucidate the exact mechanism is desirable.

sYNFACTS Contributors: Benjamin List, Lars Ratjen 